\title{
Application Research of BP neural network in face recognition
}

\author{
Yong $\mathrm{He}$ \\ Dept. of Computer Science, Xinyang Agriculture \& Forestry University, Xinyang 464000, China; \\ zzdx713hy@163.com
}

Keywords: BP neural network, face recognition, MATLAB.

\begin{abstract}
This paper introduces the design method of face recognition system is a kind of BP neural network. This design uses the forward backward propagation algorithm of multi-layer neural network, $\mathrm{BP}$ algorithm, BP neural network for face recognition was built by using MATLAB software, and the use of facial feature a large number of data for training the neural network, the neural network can distinguish different features. The experimental results show that using this method for face recognition has a good effect.
\end{abstract}

\section{Introduction}

With the development of science and technology, the development of face recognition technology is more and more urgent, such as (1) in the enterprise, residential security and management requirements, the realization of face recognition access control time and attendance system, face recognition security door etc.. (2) in the public security, judicial and criminal investigation, using face recognition, in a nationwide manhunt. (3) in the ATM bank, if the application of face recognition will be avoided because the user card and password theft. The losses caused by (4) in the process of electronic commerce transaction, if the realization of the party in the face recognition using digital identity online and true identity unity, it increases the reliability of the electronic commerce. System development of the face recognition software system is the most important link between theory and practice, so the implementation of face recognition system development application is more and more important.

\section{Organization of the Text}

BP neural network.BP (Back Propagation) neural network, which is the process of learning error back propagation of error back propagation algorithm by the reverse forward propagation and error information propagation consists of two processes. Including the input output model, function model, error model and learning model. Feedforward multilayer neural network is widely used in pattern recognition and classification, control, forecasting, the problem such as image processing, is the most widely in today's society, one of the fastest development of artificial neural network. Due to the weights of neural network in the network error Back Propagation algorithm is often used to determine the threshold (Back Propagation, referred to as BP algorithm), so usually call this feedforward network BP network. BP network is a group of scientists in 1986 by Rumelhart and McCelland led forward, is a kind of error back-propagation algorithm for training multilayer feedforward neural network, neural network model is one of the most widely used. BP network can learn and store a lot of input - output model mapping relationship, without prior to reveal the mathematical equations to describe this mapping. Its learning rule is the steepest descent method is used to continuously adjust the network weight value and threshold value by back propagation, make the network and the minimum sum of square error. BP neural network model topology structure includes input layer, hidden layer (input) (hidden layer) and the output layer (output layer).

Typical three layer BP network is a network, including the input layer, hidden layer and output layer. Practice the full connection between each layer, and the hidden layer can be a layer, can also be a multi-layer (fig. 1). 
Face recognition system.Face recognition is a biometric identification technology based on human face feature information. With the acquisition of the camera or camera with face images or video stream, and the automatic face detection and tracking in image, a series of related technology of face and then the detected face, usually also called face recognition, face recognition.

Face recognition system based on face recognition technology as the core, is an emerging biometric technology, is the key technology in the field of the international advanced technology. It is widely used in regional feature analysis algorithm, the fusion of computer image processing technology and Biostatistics at an organic whole, extraction of feature points from the video portrait by using computer image processing technology, carries on the analysis to establish the mathematical model of the use of biometrics principle, has wide development prospects. In 2012 April, the railway department announced the station security area will be installed for high-tech security system based on face recognition system identification; to face brightness detection, automatic dynamic adjustment of exposure compensation, face tracking, detection, automatic adjustment of image magnification;

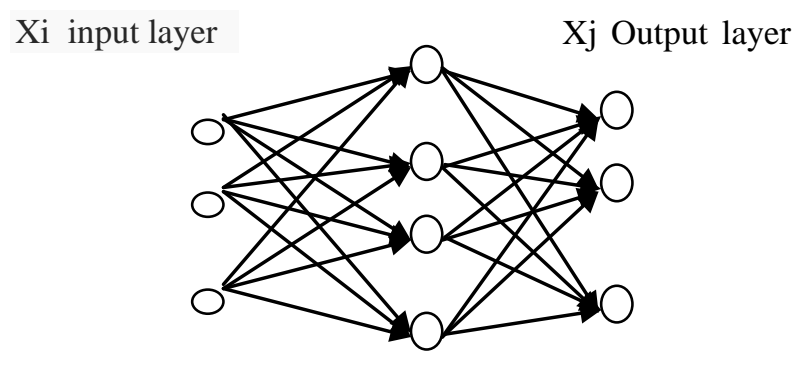

Fig. 1: a single hidden layer BP network

Face recognition system mainly consists of four parts, respectively is: face image acquisition and detection, face image preprocessing, face image feature extraction and matching and recognition.

\section{Learning of BP network is composed of four processes, namely}

(1) input mode: input layer through the hidden layer to output layer "pattern along the propagation process;

(2) the theory of network output and network: the difference between the actual output of the error signal from the output layer through the hidden layer to the input layer correction step by step a connection weight of the error back propagation process;

(3) transmitted by "model" and "error back propagation" repeatedly alternates network "memory training process;

(4) the network convergence is network global error to minimum of "learning" process. In short, is transmitted by "model" - > "error back propagation" to "memory training", "learning" process.

Transfer function is commonly $(0,1)$ model S function $f(x)=\frac{1}{1+e^{-x}}$

Neurons in the hidden layer of BP network adopts s-shaped exchange function, output layer by using pure linear transformation function.

\section{BP network algorithm}

BP algorithm can be described as roughly five steps:

(1) to initialize the random value of each weight of smaller.

(2) select the training set,

(3) based on the input output calculation theory

(4) adjust the output layer and the weights between hidden layer

(5) return to the second step is repeated until the error ideal training network. 


\section{The BP neural network to realize face recognition}

Design thinking :This design adopts the 50 images (10 individuals per 5 picture) as the training images, the application of principal component analysis to second order related training images and dimension reduction, to extract independent training base composition LianZi space structure, and the training of face image to independent base on the projection coefficient of input the improved BP neural network for training. And then to identify the face image to independent get projection coefficient based on the projection, then its input has trained BP neural network for identification. Design structure as shown in fig. 2.

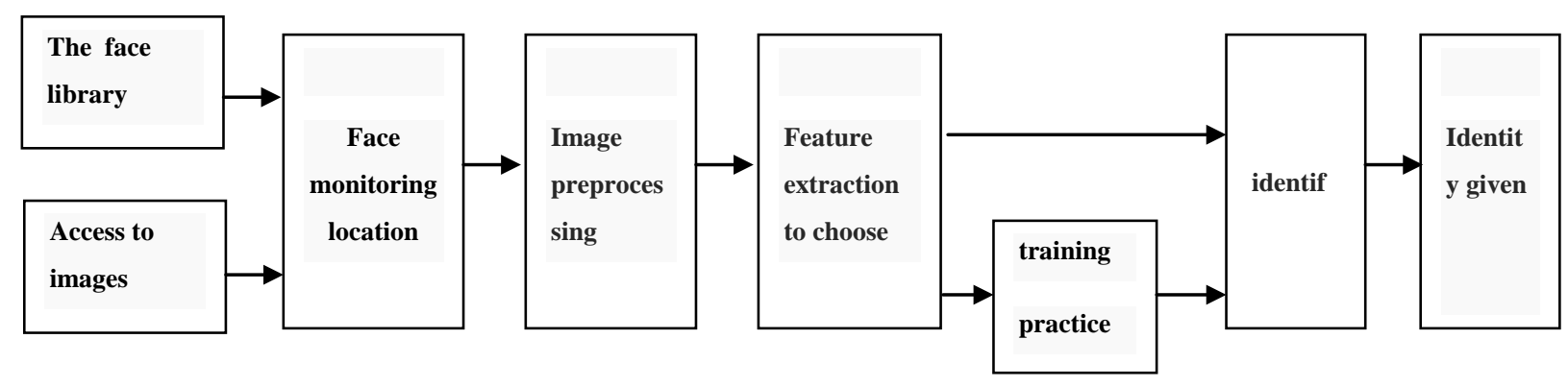

Fig.2 Face structure recognition

\section{Creation and initialization of BP network}

Because of the network input vector and output vector is large, in order to reduce the network training error, prior to establish a network to the input vector normalization. Here we use premnmx function to convert the input initialization between $[1,1]$.

\section{Training neural networks}

There are many kinds of network training function, such as, trainlm,. The traingd learning algorithm is usually the tonsure descent method; Traingdx learning algorithm is a decline in the tonsure momentum method, is adaptive learning rate. Trainlm learning algorithm for Levenberg Marquadt back propagation algorithm, the advantage of the training function convergence speed quickly. This design chooses the training function for traingdx, reach up in network training error requirement automatically stop, save training time. In the process of training to get smaller error, can increase the number of training.

\section{The system's performance evaluation}

In order to test the reliability of the network pattern recognition system design, with 50 image input samples here, to see the output results. Through the demo, basic can identify different facial features. If you want to improve the accuracy, can prolong the network training time, or network hidden layer neurons number increase. To test the system, can extract a few a few human face image data, and then put them in the input to the network, observe its output, and error analysis.

\section{Summary}

This design USES the face image data as input to train the network, using BP algorithm, by means of MATLAB software as a tool to establish face recognition of BP neural network, and using training 50 human face image data of the network, the neural network can identify the face information, improve the ability of the whole network fault tolerance, more suitable for use in a real environment. 
The result shows that this design has better advantage in face recognition. Research in face recognition of $\mathrm{BP}$ neural network, the original sample data without preprocessing and feature extraction, not only make the recognition result is accurate is reduced, and the structure of BP neural complexity. The principal component analysis method to extract a few principal components instead of samples, these principal components are uncorrelated with each other, with the characteristic of optimization requirements. Show on BioID face database experiment, combining principal component analysis and BP neural network is compared with the traditional single BP neural network recognition, can improve the correct recognition rate and reduce the training time, and simplify the network structure, reduce the amount of calculation greatly.

\section{References}

[1] L. G. wel, numerous led her, Zhang Yuan. Principal component analysis and study on the method of BP neural network face recognition [N]. The modern electronic technology, in 2007, no. 2.

[2] Martin T. Hag, Howard B.Demuth. Neural Network Design Original [M]. PSW publishing company.

[3] Zhang Wei. Introduction to MATLAB foundation and programming [M]. Xi 'an: xi 'an university of electronic science and technology press, 2011.

[4] Cong Shuang. Facing the toolbox of MATLAB neural network theory and application [M]. Hefei: university of science and technology of China press, 2012. 